\title{
The Rooted Tree Embedding Problem into Points in the Plane
}

\author{
Yoshiko Ikebe, ${ }^{1}$ Micha A. Perles, ${ }^{2}$ Akihisa Tamura, ${ }^{3}$ and Shinnichi Tokunaga ${ }^{4}$ \\ ${ }^{2}$ Department of Information Sciences, Tokyo Institute of Technology, \\ Meguro-ku, Tokyo 152, Japan \\ ikebe@is.titech.ac.jp \\ ${ }^{2}$ Department of Mathematics, The Hebrew University of Jerusalem, \\ Jerusalem, Israel \\ perles@vms.huji.ac.il \\ ${ }^{3}$ Department of Computer Science and Information Mathematics, \\ The University of Electro-Communications, Chofu, \\ Tokyo 182, Japan \\ tamura@im.uec.ac.jp \\ ${ }^{4}$ Department of Applied Mathematics, Science University of Tokyo, \\ Shinjuku-ku, Tokyo 162, Japan \\ Communicated by Imre Bárány
}

\begin{abstract}
In this paper we show that any rooted tree of $n$ vertices can be straight-line embedded into any set $S$ of $n$ points in the plane in general position so that the image of the root is arbitrarily specified.
\end{abstract}

\section{Introduction}

Let $T$ be a rooted tree with $n$ vertices and let $N$ be a set $\left\{p_{1}, p_{2}, \ldots, p_{n}\right\}$ of $n$ points in general position in $R^{2}$, i.e., no three points lie on any line. We denote the root of $T$ by $r_{1}$, and the sets of vertices and edges of $T$ by $V(T)$ and $E(T)$, respectively. We consider a bijection $\varphi$ from $V(T)$ to $N$ and define the image of each edge $u v \in E(T)$ with respect to $\varphi$ by the line segment $\overline{\varphi(u) \varphi(v)}$. Perles [3] posed the following problem.

Problem. Is there a bijection $\varphi$ from $V(T)$ to $N$ satisfying the following two conditions?

(C1) $\varphi\left(r_{1}\right)=p_{1}$.

(C2) For any two nonadjacent edges $u_{1} v_{1}, u_{2} v_{2} \in E(T)$, line segments $\overline{\varphi\left(u_{1}\right) \varphi\left(v_{1}\right)}$ and $\overline{\varphi\left(u_{2}\right) \varphi\left(v_{2}\right)}$ are disjoint. 
Here we call such a bijection a rooted tree embedding (or an rt-embedding) of $T$ on $N$.

It has been proved by Pach and Töröcsik [2] that there is an rt-embedding when the number of the minimum points of $N$ in a closed half-plane containing $p_{1}$ satisfies some specific conditions. In this paper we prove the existence of rt-embeddings by giving an algorithm that constructs one, even when $p_{1}$ does not satisfy these conditions. Furthermore, our algorithm constructs an rt-embedding in polynomial time with respect to $n$.

\section{Preliminaries}

Let $N$ be an $n$-set $\left\{p_{1}, \ldots, p_{n}\right\}$ in general position in $R^{2}$. We say that a line $l$ containing $p_{1}$ is an $(a, b)$-separator of $N-\left\{p_{1}\right\}$ if $l$ does not contain any other point of $N$, and splits $N-\left\{p_{1}\right\}$ into $a$ points and $b$ points. We also say that $l$ is an $[a, b)$-separator of $N-\left\{p_{1}\right\}$ if $l$ contains $p_{1}$ and one other point $p$, and splits $N-\left\{p_{1}, p\right\}$ into $(a-1)$ points and $b$ points. Now suppose that $l$ is a line containing $p_{1}$, such that one open half-plane determined by $l$ contains as many points of $N$ as possible. Let $t$ be the number of points of $N$ in the open half-plane and let $s=(n-1)-t$. We remark that $s$ corresponds exactly to what is called "the depth of $p_{1}$ " in [2]. Then the two open half-planes determined by any line containing $p_{1}$ contain at least $s$ points of $N-\left\{p_{1}\right\}$. We use the notations $s$ and $t$ to express these minimum and maximum numbers through this paper. The next lemmas follow from the fact that no three points of $N$ lie on any line. Proofs are omitted.

Lemma 2.1. The following statements hold.

(1) For any $j$ with $s \leq j \leq t$, there is $a(j, n-1-j)$-separator of $N-\left\{p_{1}\right\}$.

(2) For any $j$ with $s<j \leq t$, there is $a[j, n-1-j)$-separator of $N-\left\{p_{1}\right\}$.

Note that $s<j$ in (2) is the strict inequality.

Lemma 2.2. $s+1 \leq t$.

Let $T$ be a tree rooted at $r_{1}$. For each edge $u v \in E(T), u$ is called the parent of $v$ and $v$ a child of $u$ if $u$ is closer than $v$ to $r_{1}$. For any vertex $u \in V(T)$, let $D(u)$ be the set consisting of $u$ and $u$ 's descendants. We denote the number of $u$ 's children as $\operatorname{ch}(u)$. Let $\left\{v_{1}, v_{2}, \ldots, v_{\text {ch }(u)}\right\}$ be the set of $u$ 's children, and let $T^{i}(u)$ denote the subtree of $T$ induced by $D\left(v_{i}\right)$ for $i=1, \ldots, c h(u)$. We say that $v_{i}$ is the root of $T^{i}(u)$, and assume that the order of $u$ 's children is specified to satisfy the following condition:

$$
\left|T^{1}(u)\right| \geq\left|T^{2}(u)\right|, \ldots,\left|T^{c h(u)}(u)\right|
$$

where $\left|T^{i}(u)\right|$ denotes the number of vertices of $T^{i}(u)$. For convenience, we define $T^{0}(u)$ as the subtree of $T$ induced by $V(T)-D(u)$. 
We call $r_{1}$ the first master, and recursively define the $j$ th master as the first child of the $(j-1)$ th master when $j \geq 2$. The sequence $\left\{\left|T^{1}\left(r_{j}\right)\right|: j=1,2, \ldots\right\}$ is a strictly monotone decreasing sequence. Thus let $r_{k}$ be the master such that $\left|T^{1}\left(r_{k}\right)\right|<t$ and $\left|T^{1}\left(r_{k-1}\right)\right| \geq t$ if $k \geq 2$. The following lemma holds for master $r_{k}$.

Lemma 2.3. $\left|T^{0}\left(r_{k}\right)\right| \leq s+1$.

Proof. If $k=1$, then $\left|T^{0}\left(r_{1}\right)\right|=0<1 \leq s+1$. Assume that $k \geq 2$. Since $V(T)$ is partitioned into $V\left(T^{0}\left(r_{k}\right)\right)$ and $V\left(T^{1}\left(r_{k-1}\right)\right)$ and $\left|T^{1}\left(r_{k-1}\right)\right| \geq t, \quad\left|T^{0}\left(r_{k}\right)\right|=$ $n-\left|T^{1}\left(r_{k-1}\right)\right| \leq(s+t+1)-t=s+1$.

\section{Proof of the Existence of rt-Embeddings}

In this section we prove the existence of rt-embeddings. We first deal with the case where $p_{1}$ is an extreme point of the convex hull conv $(N)$ of $N$. Lemmas 3.1 and 3.2 have been discovered independently by Pach and Töröcsik [2]. Here we describe their algorithms and omit proofs.

Lemma 3.1 [2]. By using the following algorithm we can find an rt-embedding of $T$ on $N$ when $p_{1}$ is an extreme point of $\operatorname{conv}(N)$.

\section{Algorithm 1 (see Fig. 1)}

Step 1. Let $p_{2}$ be an extreme point of $\operatorname{conv}(N)$ adjacent to $p_{1}$, and create the total order $\tau$ with respect to the angle $\angle p_{2} p_{1} p$ for $p \in N-\left\{p_{1}\right\}$.

Step 2. According to the total order $\tau$ partition $N-\left\{p_{1}\right\}$ into $\operatorname{ch}\left(r_{1}\right)$ subsets $N_{1}, \ldots, N_{c h\left(r_{1}\right)}$ with $\left|N_{i}\right|=\left|T^{i}\left(r_{1}\right)\right|$, and let $p_{i}^{\prime}$ be the first point in $N_{i}$.

Step 3. Construct recursively an rt-embedding of each subtree $T^{i}\left(r_{1}\right)$ onto $N_{i}$, such that the image of the ith child of $r_{1}$ is $p_{i}^{\prime}$.

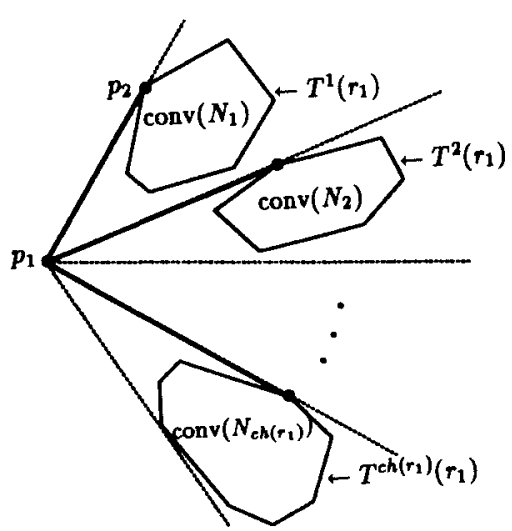

Fig. 1. Algorithm 1. 


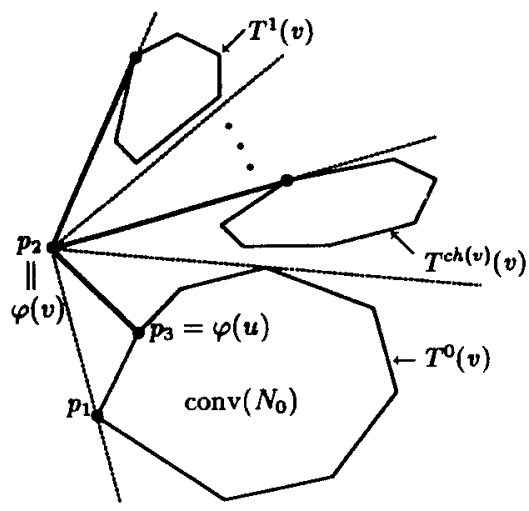

Fig. 2. Algorithm 2.

Lemma 3.2 [2]. Suppose that $p_{1}$ is an extreme point of $\operatorname{conv}(N)$ and $n \geq 2$. Let $p_{2}$ be an extreme point adjacent to $p_{1}$. Then the following algorithm constructs an rt-embedding $\varphi$ of $T$ on $N$ with $\varphi(v)=p_{2}$ for a specified vertex $v \in V(T)-\left\{r_{1}\right\}$.

\section{Algorithm 2 (see Fig. 2)}

Step 1. If $v$ is a child of $r_{1}$, then use Algorithm 1 to find an rt-embedding of $T$ on $N$, otherwise create the total order $\tau$ with respect to the angle $\angle p_{1} p_{2} p$ for $p \in N-\left\{p_{2}\right\}$.

Step 2. Let $N_{0}$ be the first $\left|T_{0}(v)\right|$ points of $N$ with respect to $\tau ; N_{1}=N-N_{0}$; and let $p_{3}$ be an extreme point of $\operatorname{conv}\left(N_{0}\right)$ adjacent to $p_{1}$ and visible from $p_{2}$.

Step 3. Construct recursively an rt-embedding of $T^{0}(v)$ on $N_{0}$ such that $v$ 's parent $u$ is mapped to $p_{3}$; use Algorithm 1 to construct an rt-embedding of $T(v)$ on $N_{1}$ such that $v$ is mapped to $p_{2}$

In the rest of the paper we ignore trivial cases when $n \leq 2$. Recall that $r_{k}$ is the master such that

$$
\left[\left|T^{1}\left(r_{k}\right)\right|<t\right] \text { and } \quad\left[\left|T^{1}\left(r_{k-1}\right)\right| \geq t \text { if } k \geq 2\right]
$$

Now we consider the following three cases for the master $r_{k}$.

Case 1: $k=1$ (in other cases we assume $k \geq 2$ ).

Case 2: $h \in\left\{1, \ldots, \operatorname{ch}\left(r_{k}\right)\right\}$ exists with $s \leq\left|T^{1}\left(r_{k}\right)\right|+\cdots+\left|T^{h}\left(r_{k}\right)\right|<t$.

Case 3: otherwise.

Note that Case 1 contains the case when $p_{1}$ is an extreme point of $\operatorname{conv}(N)$. Now 
suppose that $k \geq 2$. From the definitions of $s, t$, and $T^{i}\left(r_{k}\right), \sum_{i=0}^{c h\left(r_{k}\right)}\left|T^{i}\left(r_{k}\right)\right|+1=$ $n=s+t+1$ and $\left|T^{0}\left(r_{k}\right)\right| \leq s+1$ (Lemma 2.3). If $s \leq\left|T^{1}\left(r_{k}\right)\right|$, then we have Case 2. On the other hand if $\left|T^{0}\left(r_{k}\right)\right|=s+1$, then $s \leq\left|T^{1}\left(r_{k}\right)\right|+\cdots+\left|T^{c h\left(r_{k}\right)}\left(r_{k}\right)\right|=t-1$ (the inequality follows from Lemma 2.2), and again we have Case 2. Thus, from Lemma 2.3 and assumptions (2.1) and (3.1), the following conditions hold in Case 3 :

$$
\left|T^{0}\left(r_{k}\right)\right| \leq s \quad \text { and } \quad\left|T^{i}\left(r_{k}\right)\right|<s \quad \text { for } \quad i=1,2, \ldots, \operatorname{ch}\left(r_{k}\right)
$$

We first prove that there is an rt-embedding in Cases 1 and 2. We add here that Pach and Töröcsik, by using a different partitioning of $T$, have given a similar proof for what is essentially Case 2 and part of Case 1 in [2].

Lemma 3.3. In Case 1 there is an rt-embedding of $T$ on $N$.

Proof. We first consider the case when $s \leq\left|T^{1}\left(r_{1}\right)\right|$. From (1) of Lemma 2.1, we can use a clockwise ordering around $p_{1}$ to partition $N-N_{1}$ so that it satisfies

$$
\operatorname{conv}\left(N_{i} \cup\left\{p_{1}\right\}\right) \cap \operatorname{conv}\left(N_{j} \cup\left\{p_{1}\right\}\right)=\left\{p_{1}\right\} \quad \text { if } \quad i \neq j
$$

(see Fig. 3). Since $p_{1}$ is an extreme point of the convex hull of $N_{i} \cup p_{1}$ for each $i$, we can use Algorithm 1 to construct an rt-embedding of $T$ on $N$.

If $\left|T^{1}\left(r_{1}\right)\right|<s$, then $\left|T^{i}\left(r_{1}\right)\right|<s$ for all $i$ from assumption (2.1). Let $p_{2}$ be any point of $N-\left\{p_{1}\right\}$. In the same manner as above, we partition $N$ into $\left\{N_{1}, \ldots, N_{\text {ch }\left(r_{1}\right)}\right\}$ with $\left|N_{i}\right|=\left|T^{i}\left(r_{1}\right)\right|$ for $i=1, \ldots, \operatorname{ch}\left(r_{1}\right)$ according to a clockwise ordering on $N-\left\{p_{1}\right\}$ around $p_{1}$ beginning from $p_{2}$ (see Fig. 4). Since $\left|T^{i}\left(r_{1}\right)\right|<s$ for each $i$, the partition satisfies (3.3). Thus, we can construct an rt-embedding of $T$ on $N$.

Lemma 3.4. In Case 2 there is an rt-embedding of $T$ on $N$.

Proof. Let $T^{1}$ and $T^{0}$ denote the subtrees of $T$ induced by

$$
V\left(T^{1}\left(r_{k}\right)\right) \cup \cdots \cup V\left(T^{h}\left(r_{k}\right)\right) \cup\left\{r_{k}\right\}
$$

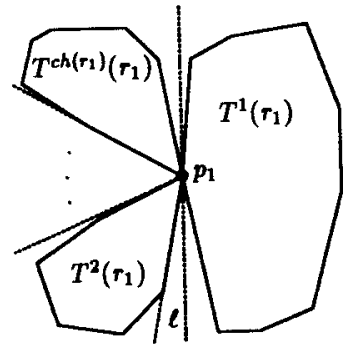

Fig. 3. Case 1.

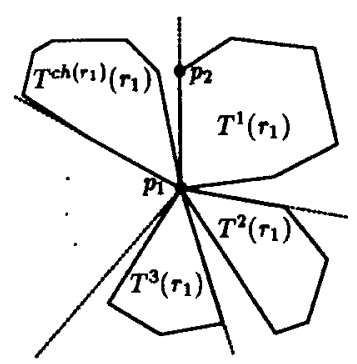

Fig. 4. Case 1. 


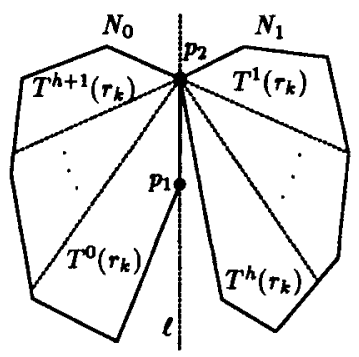

Fig. 5. Case 2.

and $V\left(T^{0}\left(r_{k}\right)\right) \cup V\left(T^{h+1}\left(r_{k}\right)\right) \cup \cdots \cup V\left(T^{c h\left(r_{k}\right)}\left(r_{k}\right)\right) \cup\left\{r_{k}\right\}$, respectively. Then $E(T)$ is partitioned into $E\left(T^{1}\right)$ and $E\left(T^{0}\right)$. From (2) of Lemma 2.1, there is a $\left[\left|T^{1}\right|, n-1-\left|T^{1}\right|\right)$-separator $l$ of $N-\left\{p_{1}\right\}$ because $s<\left|T^{1}\right| \leq t$. Suppose that $p_{2}$ is the point distinct from $p_{1}$ on $l$. Let $N_{1}$ be the subset of $N$ consisting of $p_{2}$ and the $\left(\left|T^{1}\right|-1\right)$ points in an open half-plane determined by $l$, and let $N_{1}$ be the points of $N$ in the opposite closed half-plane (see Fig. 5). Then $\left|N_{0}\right|=\left|T^{0}\right|,\left|N_{1}\right|=\left|T^{1}\right|$, and $\operatorname{conv}\left(N_{0}\right) \cap \operatorname{conv}\left(N_{1}\right)=\left\{p_{2}\right\}$. Since $p_{2}$ is an extreme point of conv $\left(N_{0}\right)$ adjacent to $p_{1}$, we may construct an rt-embedding $\varphi_{0}$ of $T^{0}$ on $N_{0}$ with $\varphi_{0}\left(r_{k}\right)=p_{2}$ by using Algorithm 2. On the other hand, by using Algorithm 1 we may construct an rt-embedding $\varphi_{1}$ of $T^{1}$ on $N_{1}$ with $\varphi_{1}\left(r_{k}\right)=p_{2}$ because $p_{2}$ is an extreme point of $\operatorname{conv}\left(N_{1}\right)$. Then the bijection $\varphi$ from $V(T)$ to $N$ defined by $\varphi_{0}$ and $\varphi_{1}$ is an rt-embedding of $T$ on $N$.

Before discussing the proof for Case 3, we give some definitions and a lemma. In Case 3 the inequality $t \leq\left|T^{1}\left(r_{k}\right)\right|+\cdots+\left|T^{c h\left(r_{k}\right)}\left(r_{k}\right)\right|$ holds; from (3.1) the right-hand side is greater than or equal to $s$ and if it is less than $t$, then we would have Case 2. Therefore, there is an $h \in\left\{2, \ldots, c h\left(r_{k}\right)\right\}$ such that

$$
\left|T^{1}\left(r_{k}\right)\right|+\cdots+\left|T^{h-1}\left(r_{k}\right)\right|<s<t \leq\left|T^{1}\left(r_{k}\right)\right|+\cdots+\left|T^{h}\left(r_{k}\right)\right|
$$

Let $T^{0}$ and $T^{1}$ be the subtrees of $T$ induced by $V\left(T^{0}\right)$ and $V\left(T^{1}\right)$ defined as

$$
\begin{aligned}
& V\left(T^{0}\right)=\left\{r_{k}\right\} \cup V\left(T^{h+1}\left(r_{k}\right)\right) \cup \cdots \cup V\left(T^{c h\left(r_{k}\right)}\left(r_{k}\right)\right) \cup V\left(T^{0}\left(r_{k}\right)\right), \\
& V\left(T^{1}\right)=\left\{r_{k}\right\} \cup V\left(T^{1}\left(r_{k}\right)\right) \cup \cdots \cup V\left(T^{h-1}\left(r_{k}\right)\right) .
\end{aligned}
$$

From the above definitions, $E(T)$ is partitioned into $E\left(T^{0}\right), E\left(T^{1}\right), E\left(T^{h}\left(r_{k}\right)\right)$, and the edge $r_{k} r$, where $r$ is the root of $T^{h}\left(r_{k}\right)$ (see Fig. 6). We obtain the next lemma. 


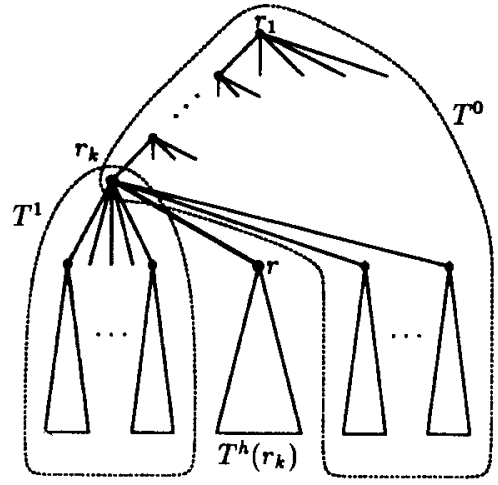

Fig. 6. Decomposition of tree.

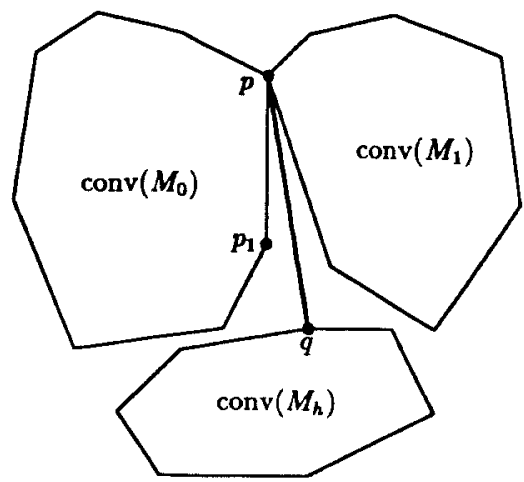

Fig. 7. Decomposition of points.

Lemma 3.5. If there are subsets $M_{0}, M_{1}, M_{h}$ of $N$ and points $p, q \in N$ such that

$$
\begin{aligned}
\left|M_{0}\right|=\left|T^{0}\right|, \quad\left|M_{1}\right|=\left|T^{1}\right|, \quad\left|M_{h}\right|=\left|T^{h}\left(r_{k}\right)\right|, \\
\operatorname{conv}\left(M_{0}\right) \cap \operatorname{conv}\left(M_{1}\right)=\{p\}, \\
\operatorname{conv}\left(M_{0}\right) \cap \operatorname{conv}\left(M_{h}\right)=\varnothing, \\
\operatorname{conv}\left(M_{1}\right) \cap \operatorname{conv}\left(M_{h}\right)=\varnothing, \\
\operatorname{conv}\left(M_{0}\right) \cap \overline{p q}=\{p\}, \\
\operatorname{conv}\left(M_{1}\right) \cap \overline{p q}=\{p\}, \\
\operatorname{conv}\left(M_{k}\right) \cap \overline{p q}=\{q\}
\end{aligned}
$$

$p$ and $p_{1}$ are extreme points of conv $\left(M_{0}\right)$ adjacent to each other,

then there is an rt-embedding of $T$ on $N$ (see Fig. 7).

Proof. We may argue in the following way from the fact that $N$ is in general position. From assumption (3.6), $p$ is an extreme point of $\operatorname{conv}\left(M_{0}\right)$, also of conv $\left(M_{1}\right)$. Then there are rt-embeddings $\varphi_{0}$ of $T^{0}$ on $M_{0}$ and $\varphi_{1}$ of $T^{1}$ on $M_{1}$ with $\varphi_{0}\left(r_{k}\right)=\varphi_{1}\left(r_{k}\right)=p$, by Lemmas 3.1 and 3.2 and by assumptions (3.5) and (3.12). Since $q$ is an extreme point of conv $\left(M_{h}\right)$ from (3.11), an rt-embedding $\varphi_{h}$ of $T^{h}\left(r_{k}\right)$ on $M_{h}$ exists with $\varphi_{h}(r)=q$ where $r$ is the root of $T^{h}\left(r_{k}\right)$, by Lemma 3.1 and assumption (3.5). Then the bijection from $V(T)$ to $N$ defined by $\varphi_{0}, \varphi_{1}$, and $\varphi_{h}$ is an rt-embedding of $T$ on $N$ by assumptions (3.6)-(3.11). 
In order to prove the existence of rt-embeddings, it is enough to show that $N$ can be distributed so that all the conditions in the above lemma hold.

We select any point $p_{2}$ from $N-\left\{p_{1}\right\}$ so that the line $l\left(p_{1} p_{2}\right)$ passing through $p_{1}$ and $p_{2}$ is an $[s+1, t-1)$-separator. Without loss of generality, assume that $p_{2}$ is directly above $p_{1}$ and that the right-hand side of the line belongs to $s$ points of $N$. Let $\tau$ be a clockwise ordering on $N-\left\{p_{1}\right\}$ around $p_{1}$ beginning from $p_{2}$. According to this total order $\tau$, we can partition $N-\left\{p_{1}, p_{2}\right\}$ into three subsets $\left\{N_{1}, N_{h}, N_{0}\right\}$ such that $\left|N_{1}\right|=\left|T^{1}\right|-1,\left|N_{h}\right|=\left|T^{h}\left(r_{k}\right)\right|$, and $\left|N_{0}\right|=\left|T^{0}\right|-2$ because $\left|T^{1}\right|+\left|T^{h}\left(r_{k}\right)\right|+\left|T^{0}\right|=n+1$. Let $M_{0}=N_{0} \cup\left\{p_{1}, p_{2}\right\}, M_{1}=N_{1} \cup\left\{p_{2}\right\}$, and $M_{h}=N_{h}$. Then (3.5) holds for $M_{0}, M_{1}$, and $M_{h}$. We write the first and last points of $N_{h}$ with respect to $\tau$ as $p_{3}$ and $p_{4}$, respectively. From (3.2) and (3.4), angles $\angle p_{2} p_{1} p_{3}, \angle p_{2} p_{1} p_{4}$, and $\angle p_{3} p_{1} p_{4}$ are less than $\pi$. More precisely, the following relations hold:

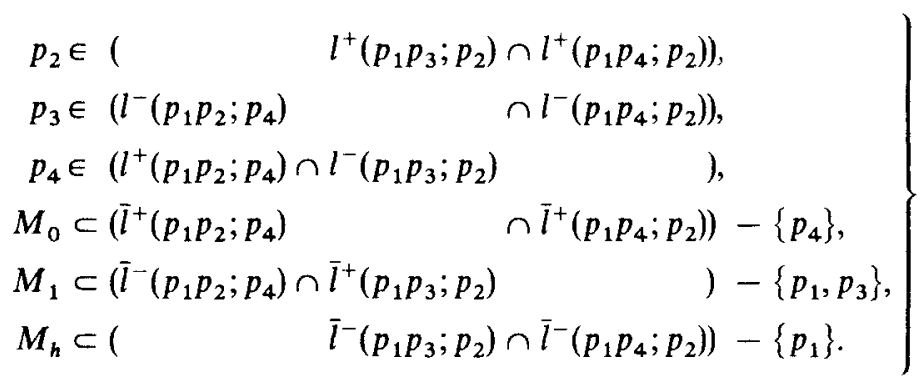

Here, for distinct points $p, q, r \in N$, we write the closed (or open) half-plane determined by the line $l(p q)$ including $r$ as $\bar{l}^{+}(p q ; r)$ (or $l^{+}(p q ; r)$ ) and the opposite closed (or open) half-plane as $\bar{l}^{-}(p q ; r)$ (or $\left.l^{-}(p q ; r)\right)$. From (3.13), it follows that conditions (3.6) $-(3.9)$ and condition (3.12) hold by setting $p=p_{2}$ and $q=p_{3}$. The definition of $p_{3}$ implies (3.11). Thus the sets $M_{0}, M_{1}, M_{h}$ and the points $p_{2}, p_{3}$ satisfy all the conditions in Lemma 3.5 other than (3.10). When these do not satisfy (3.10), they will be improved to do so.

Let $q_{2}$ be the extreme point of $\operatorname{conv}\left(M_{1}\right)$ which is next to $p_{2}$ with respect to the counterclockwise ordering on the extreme points of $\operatorname{conv}\left(M_{1}\right)$. We consider three subcases of Case 3.

Case 3.1: $p_{3} \in l^{+}\left(p_{2} q_{2} ; p_{1}\right)$ (Fig. 8).

Case 3.2: $p_{3} \in l^{-}\left(p_{2} q_{2} ; p_{1}\right)$ and $q_{2} \in l^{-}\left(p_{1} p_{4} ; p_{2}\right)$ (Fig. 9).

Case 3.3: $p_{3} \in l^{-}\left(p_{2} q_{2} ; p_{1}\right)$ and $q_{2} \in l^{+}\left(p_{1} p_{4} ; p_{2}\right)$ (Fig. 10).

We show the existence of an rt-embedding of $T$ on $N$ in each case.

Lemma 3.6. In Case 3.1 there is an rt-embedding of $T$ on $N$.

Proof. From the definition of $q_{2}, M_{1} \in \bar{l}^{-}\left(p_{2} q_{2} ; p_{1}\right)$. The assumption of Case 3.1 implies (3.10). From the above discussion, the assertion follows from Lemma 3.5. 


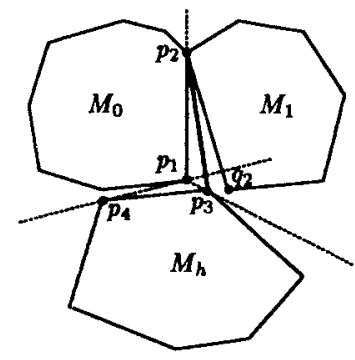

Fig. 8. Case 3.1.

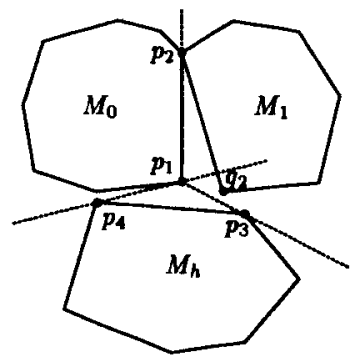

Fig. 9. Case 3.2 .

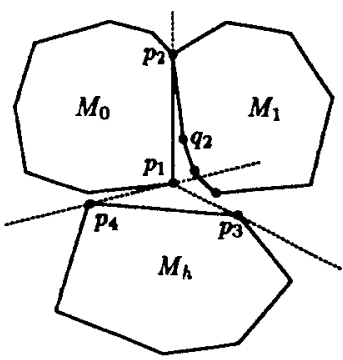

Fig. 10. Case 3.3 .

Lemma 3.7. In Case 3.2 there is an rt-embedding of $T$ on $N$.

Proof. Figures 11 and 12 may help the reader to understand this proof. In this case $M_{1}, p_{2}$, and $p_{3}$ do not satisfy (3.10). Suppose that $p$ is the point of $\left(M_{1} \cup M_{h}\right)-\left\{q_{2}\right\}$ which is the $\left|M_{1}\right|$ th point among $\left(M_{1} \cup M_{h}\right)-\left\{q_{2}\right\}$ with respect to the total order determined by angles $\angle p_{2} q_{2} q$ for $q \in\left(M_{1} \cup M_{h}\right)-\left\{q_{2}\right\}$. Let $M_{1}^{\prime}$ be the set of the first $\left|M_{1}\right|$ points of $\left(M_{1} \cup M_{h}\right)-\left\{q_{2}\right\}$ and let $M_{h}^{\prime}=$ $\left(M_{1} \cup M_{h}\right)-M_{1}^{\prime}$.

Roughly speaking, the plane is partitioned into four convex regions: the triangle $\Delta p_{1} p_{2} q_{2}$, above the polygonal line $p_{4} p_{1} p_{2}$, below the polygonal line $p_{4} p_{1} q_{2} p$, and the rest. The last three unbounded regions include $M_{0}, M_{h}^{\prime}$, and $M_{1}^{\prime}$, respectively. More precisely, the following relations hold:

$$
\begin{aligned}
& \overline{p_{2} q_{2}} \subset\left(\bar{l}^{-}\left(p_{1} p_{2} ; p_{4}\right)\right. \\
& \left.\cap \bar{l}^{+}\left(p_{2} q_{2} ; p_{1}\right) \cap \bar{l}^{+}\left(p_{1} q_{2} ; p_{2}\right)\right) \text {, } \\
& M_{0} \subset\left(\bar{l}^{+}\left(p_{1} p_{2} ; p_{4}\right) \cap \bar{l}^{+}\left(p_{1} p_{4} ; p_{2}\right)\right. \\
& M_{1}^{\prime} \subset\left(\bar{l}^{-}\left(p_{1} p_{2} ; p_{4}\right)\right. \\
& \cap \bar{l}^{+}\left(q_{2} p ; p_{2}\right) \cap \bar{l}^{-}\left(p_{2} q_{2} ; p_{1}\right) \\
& )-\left\{p_{4}\right\}, \\
& M_{h}^{\prime} \subset \text { ( } \\
& \bar{l}^{-}\left(p_{1} p_{4} ; p_{2}\right) \cap \bar{l}^{-}\left(q_{2} p ; p_{2}\right) \\
& )-\left\{p_{1}, q_{2}\right\}, \\
& \left.\cap \bar{l}^{-}\left(p_{1} q_{2} ; p_{2}\right)\right)-\left\{p_{1}, p\right\} .
\end{aligned}
$$

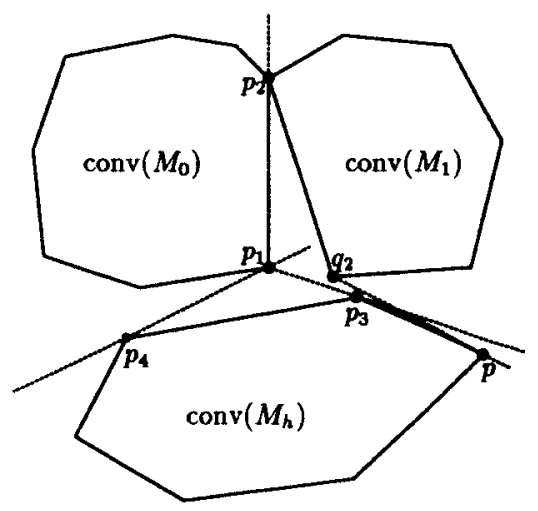

Fig. 11. Case 3.2 .

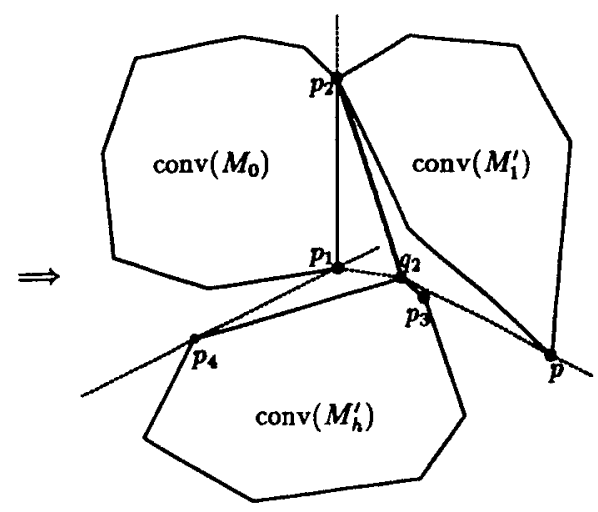

Fig. 12. New partition: $M_{1}^{\prime}$ and $M_{h}^{\prime}$. 
Hence $M_{0}, M_{1}^{\prime}, M_{h}^{\prime}, p_{2}$, and $q_{2}$ satisfy conditions (3.5)-(3.12). By Lemma 3.5, there is an rt-embedding of $T$ on $N$.

In the rest of the proof we prove the above relations. Since $M_{1} \cup\left\{p_{3}\right\} \subset$ $\bar{l}^{-}\left(p_{1} p_{2} ; p_{4}\right) \cap \bar{l}^{-}\left(p_{2} q_{2} ; p_{1}\right), \quad M_{1}^{\prime} \subset \bar{l}^{-}\left(p_{1} p_{2} ; p_{4}\right) \cap \bar{l}^{-}\left(p_{2} q_{2} ; p_{1}\right) \cap \bar{l}^{+}\left(q_{2} p ; p_{2}\right)$ and $p \in l^{-}\left(p_{2} q_{2} ; p_{1}\right)$. The assumption that $q_{2} \in l^{-}\left(p_{1} p_{4} ; p_{2}\right)$ and the fact that $q_{2} \in l^{+}\left(p_{1} p_{3} ; p_{2}\right)$ imply that $M_{h} \subset l^{-}\left(p_{1} q_{2} ; p_{2}\right) \cap l^{-}\left(p_{1} p_{4} ; p_{2}\right)$. Then $p \in l^{-}\left(p_{1} q_{2} ; p_{2}\right)$ because $\left|\left(M_{1} \cup M_{h}\right) \cap l^{+}\left(p_{1} q_{2} ; p_{2}\right)\right|<\left|M_{1}\right|$. Since $p \in l^{-}\left(p_{1} q_{2} ; p_{2}\right) \cap l^{-}\left(p_{2} q_{2} ; p_{1}\right)$, $p_{1} \in l^{-}\left(q_{2} p ; p_{2}\right)$ holds. From the definition of $M_{h}^{\prime}$,

$$
M_{h}^{\prime} \subset \bar{l}^{-}\left(p_{1} q_{2} ; p_{2}\right) \cap \bar{l}^{-}\left(p_{1} p_{4} ; p_{2}\right) \cap \bar{l}^{-}\left(q_{2} p ; p_{2}\right)
$$

because $M_{h} \subset l^{-}\left(p_{1} q_{2} ; p_{2}\right) \cap \bar{l}^{-}\left(p_{1} p_{4} ; p_{2}\right)$ and $p_{1} \in l^{-}\left(q_{2} p ; p_{2}\right)$. To summarize the discussion, the above relations can be shown.

Lemma 3.8. In Case 3.3 there is an rt-embedding of $T$ on $N$.

Proof. We first modify the sets $M_{0}$ and $M_{1}$ (see Figs. 13 and 14). Now we consider the counterclockwise sequence $\sigma$ of extreme points of $\operatorname{conv}\left(M_{1}\right)$ beginning from $p_{2}$. Since $\left|M_{h}\right|<s$, there is at least one extreme point of $\operatorname{conv}\left(M_{1}\right)$ in $l^{-}\left(p_{1} p_{4} ; p_{2}\right)$. Let $q_{3}^{\prime}$ be the first point in $\sigma$ with $q_{3}^{\prime} \in I^{-}\left(p_{1} p_{4} ; p_{2}\right)$, and let $q_{2}^{\prime}$ be the previous point of $q_{3}^{\prime}$ in $\sigma$. Suppose that $\tau$ is the clockwise ordering on $\left(M_{0} \cup M_{1}\right)-\left\{q_{2}^{\prime}\right\}$ around $q_{2}^{\prime}$ beginning from $p_{1}$. Let $M_{0}^{\prime}$ be the set which consists of $q_{2}^{\prime}$ and the first $\left(\left|M_{0}\right|-1\right)$ points of $\left(M_{0} \cup M_{1}\right)-\left\{q_{2}^{\prime}\right\}$ with respect to $\tau$, and let $M_{1}^{\prime}$ be the set consisting of $q_{2}^{\prime}$ and the last $\left(\left|M_{1}\right|-1\right)$ points with respect to $\tau$. Then $M_{0}^{\prime} \cup M_{1}^{\prime}=M_{0} \cup M_{1}$ and the first point $p^{\prime}$ among $M_{1}^{\prime}$ is the $\left|M_{0}\right|$ th point among $\left(M_{0} \cup M_{1}\right)-\left\{q_{2}^{\prime}\right\}$. As in Fig. 14, $M_{0}^{\prime}$ and $M_{1}^{\prime}$ are included in two convex regions: the left-hand side of the polygonal line $p^{\prime} q_{2}^{\prime} p_{1} p_{4}$ and the right-hand side of the polygonal line $p^{\prime} q_{2}^{\prime} q_{3}^{\prime}$, respectively. We first prove this.

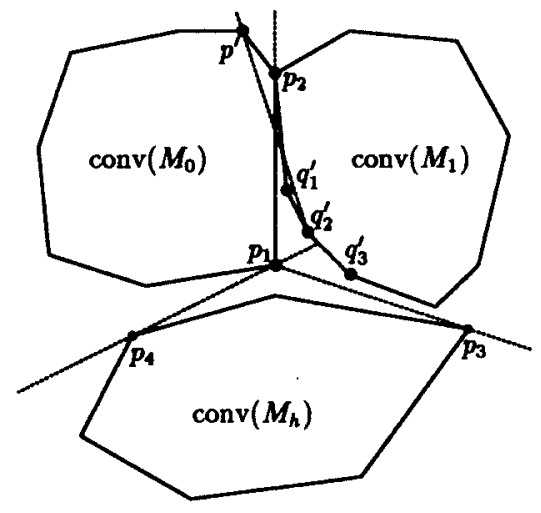

Fig. 13. Case 3.3.

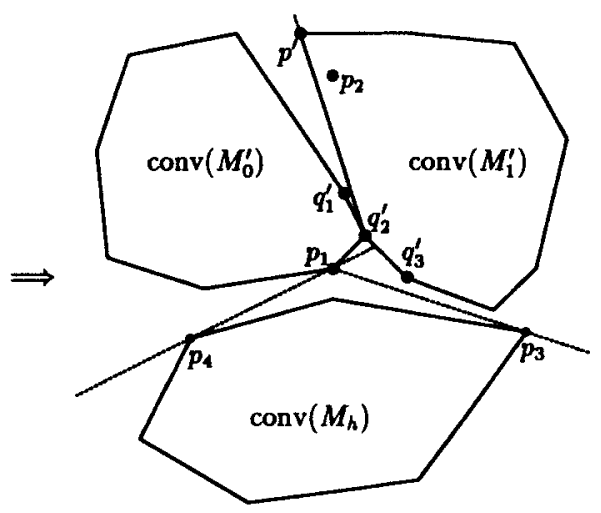

Fig. 14. New partition: $M_{0}^{\prime}$ and $M_{1}^{\prime}$. 
The point $q_{2}^{\prime}$ is distinct from $p_{2}$ because of the assumption of Case 3.3, that is, $q_{2}^{\prime} \in l^{-}\left(p_{1} p_{2} ; p_{4}\right)$. Furthermore, $q_{2}^{\prime}$ belongs to $l^{+}\left(p_{1} p_{4} ; p_{2}\right)$ and to $l^{+}\left(p_{1} p_{3} ; p_{2}\right)$. This implies $\overline{q_{2}^{\prime} p_{3}} \in \bar{l}^{-}\left(p_{1} q_{2}^{\prime} ; p_{4}\right) \cap \bar{l}^{+}\left(p_{1} p_{3} ; p_{2}\right)$. The fact that

$$
q_{2}^{\prime} \in l^{+}\left(p_{1} p_{4} ; p_{2}\right) \cap \vec{l}^{-}\left(p_{1} p_{2} ; p_{4}\right)
$$

implies $M_{0} \subset \bar{l}^{+}\left(p_{1} p_{4} ; p_{2}\right) \cap \bar{l}^{+}\left(p_{1} q_{2}^{\prime} ; p_{4}\right)$. Since $\bar{l}^{+}\left(p_{1} p_{4} ; p_{2}\right) \cap \bar{l}^{+}\left(p_{1} q_{2}^{\prime} ; p_{4}\right)$ contains at least $\left|M_{0}\right|$ points of $\left(M_{0} \cup M_{1}\right)-\left\{q_{2}^{\prime}\right\}, p^{\prime} \in l^{+}\left(p_{1} q_{2}^{\prime} ; p_{4}\right)$ and $M_{0}^{\prime} \subset$ $\bar{l}^{+}\left(p_{1} p_{4} ; p_{2}\right) \cap \bar{l}^{+}\left(p_{1} q_{2}^{\prime} ; p_{4}\right) \cap \bar{l}^{+}\left(q_{2}^{\prime} p^{\prime} ; p_{1}\right)$. For the previous point $q_{1}^{\prime}$ of $q_{2}^{\prime}$ in $\sigma$, the open half-plane $l^{+}\left(q_{2}^{\prime} q_{1}^{\prime} ; p_{1}\right)$ does not contain any point of $M_{1}$, particularly, does not contain $p_{2}$. Then $\bar{l}^{+}\left(p_{1} q_{2}^{\prime} ; p_{4}\right) \cap l^{+}\left(q_{2}^{\prime} q_{1}^{\prime} ; p_{1}\right)$ contains at most $\left(\left|M_{0}\right|-1\right)$ points of $\left(M_{0} \cup M_{1}\right)-\left\{q_{2}^{\prime}\right\}$. Hence $p^{\prime}$ belongs to $\bar{l}^{-}\left(q_{2}^{\prime} q_{1}^{\prime} ; p_{1}\right)$. The angle $\angle p^{\prime} q_{2}^{\prime} q_{3}^{\prime}$ is less than $\pi$. Thus $M_{1}^{\prime} \subset \bar{l}^{-}\left(q_{2}^{\prime} p^{\prime} ; p_{1}\right) \cap \bar{l}^{-}\left(q_{2}^{\prime} q_{3}^{\prime} ; p_{1}\right)$.

We consider two cases: the former (Case 3.3(a)) assumes $M_{h} \subset l^{+}\left(q_{2}^{\prime} q_{3}^{\prime} ; p_{1}\right)$ and the latter (Case 3.3(b)) assumes $M_{h} \not \subset l^{+}\left(q_{2}^{\prime} q_{3}^{\prime} ; p_{1}\right)$.

Case 3.3(a). The above discussion says that

$$
\begin{aligned}
& \overline{q_{2}^{\prime} p_{3}} \subset\left(\quad \bar{l}^{+}\left(q_{2}^{\prime} q_{3}^{\prime} ; p_{1}\right) \cap \bar{l}^{-}\left(p_{1} q_{2}^{\prime} ; p_{4}\right) \cap \bar{l}^{+}\left(p_{1} p_{3} ; p_{2}\right)\right), \\
& M_{0}^{\prime} \subset\left(\bar{l}^{+}\left(p_{1} p_{4} ; p_{2}\right) \cap \bar{l}^{+}\left(q_{2}^{\prime} p^{\prime} ; p_{1}\right) \quad \cap \bar{l}^{+}\left(p_{1} q_{2}^{\prime} ; p_{4}\right) \quad\right)-\left\{p_{4}, p^{\prime}\right\}, \\
& M_{1}^{\prime} \subset\left(\quad \bar{l}^{-}\left(q_{2}^{\prime} p^{\prime} ; p_{1}\right) \cap \bar{l}^{-}\left(q_{2}^{\prime} q_{3}^{\prime} ; p_{1}\right)\right. \\
& \left.M_{h} \subset \bar{l}^{-}\left(p_{1} p_{4} ; p_{2}\right) \quad \cap l^{+}\left(q_{2}^{\prime} q_{3}^{\prime} ; p_{1}\right) \quad \cap \bar{l}^{-}\left(p_{1} p_{3} ; p_{2}\right)\right)-\left\{p_{1}\right\} \text {. }
\end{aligned}
$$

These relations guarantee that $M_{0}^{\prime}, M_{1}^{\prime}, M_{h}, q_{2}^{\prime}$, and $p_{3}$ satisfy all conditions in Lemma 3.5.

Case 3.3(b). In this case there is an instance such that $M_{0}^{\prime}, M_{1}^{\prime}, M_{h}, q_{2}^{\prime}$, and $p_{3}$ satisfy all the conditions in Lemma 3.5. However, we modify $M_{1}^{\prime}$ and $M_{h}$ in order that all the conditions in Lemma 3.5 hold (see Figs. 15 and 16). Let $p^{\prime \prime}$ be the $\left|M_{1}^{\prime}\right|$ th

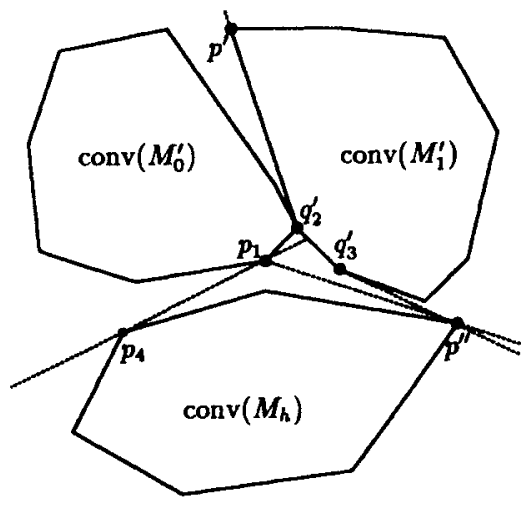

Fig. 15. Case 3.3(b).

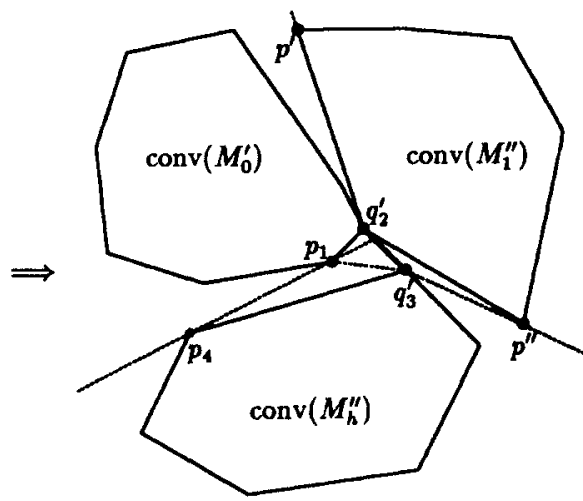

Fig. 16. New partition: $M_{1}^{\prime \prime}$ and $M_{h}^{\prime \prime}$. 
point among $\left(M_{1}^{\prime} \cup M_{h}\right)-\left\{q_{3}^{\prime}\right\}$ with respect to the clockwise ordering $\tau^{\prime}$ on $\left(M_{1}^{\prime} \cup M_{h}\right)-\left\{q_{3}^{\prime}\right\}$ around $q_{3}^{\prime}$ beginning from $q_{2}^{\prime}$. Let $M_{1}^{\prime \prime}$ be the first $\left|M_{1}^{\prime}\right|$ points among $\left(M_{1}^{\prime} \cup M_{h}\right)-\left\{q_{3}^{\prime}\right\}$ with respect to $\tau^{\prime}$ and let $M_{h}^{\prime \prime}=\left(M_{1}^{\prime} \cup M_{h}\right)-M_{1}^{\prime \prime}$. The set $M_{h}^{\prime \prime}$ contains $q_{3}^{\prime}$. Then the plane is partitioned into four convex regions: the triangle $\Delta p_{1} q_{2}^{\prime} q_{3}^{\prime}$, the left-hand side of the polygonal line $p^{\prime} q_{2}^{\prime} p_{1} p_{4}$, the right-hand side of the polygonal line $p^{\prime} q_{2}^{\prime} q_{3}^{\prime} p^{\prime \prime}$, and below the polygonal line $p_{4} p_{1} q_{3}^{\prime} p^{\prime \prime}$. The last three unbounded regions include $M_{0}^{\prime}, M_{1}^{\prime \prime}$, and $M_{h}^{\prime \prime}$, respectively (see Fig. 16). We prove this fact below.

Since $q_{3}^{\prime} \in l^{-}\left(p_{1} p_{4} ; p_{2}\right) \cap l^{+}\left(p_{1} p_{3} ; p_{2}\right), M_{h} \subset l^{-}\left(p_{1} q_{3}^{\prime} ; q_{2}^{\prime}\right)$. Then $p^{\prime \prime} \in l^{-}\left(p_{1} q_{3}^{\prime} ; q_{2}^{\prime}\right)$ because $\left|\left(M_{1}^{\prime} \cup M_{h}\right) \cap l^{+}\left(p_{1} q_{3}^{\prime} ; q_{2}^{\prime}\right)\right|<\left|M_{1}^{\prime}\right|$. Since

$$
M_{h} \subset \bar{l}^{-}\left(p_{1} p_{4} ; p_{2}\right) \cap \bar{l}^{-}\left(p_{1} q_{3}^{\prime} ; q_{2}^{\prime}\right)
$$

and $p^{\prime \prime} \in l^{-}\left(p_{1} q_{3}^{\prime} ; q_{2}^{\prime}\right)$, the definition of $M_{h}^{\prime \prime}$ implies

$$
M_{h}^{\prime \prime} \subset \bar{l}^{-}\left(p_{1} p_{4} ; p_{2}\right) \cap \bar{l}^{-}\left(p_{1} q_{3}^{\prime} ; q_{2}^{\prime}\right) \cap \bar{l}^{+}\left(q_{3}^{\prime} p^{\prime \prime} ; p_{1}\right)
$$

From the assumption of Case 3.3(b), $\bar{l}^{-}\left(q_{2}^{\prime} q_{3}^{\prime} ; p_{1}\right)$ contains at least $\left(\left|M_{1}^{\prime}\right|+1\right)$ points of $M_{1}^{\prime} \cup M_{h}$. Then $p^{\prime \prime} \in l^{-}\left(q_{2}^{\prime} q_{3}^{\prime} ; p_{1}\right)$ and

$$
M_{1}^{\prime \prime} \subset \bar{l}^{-}\left(q_{2}^{\prime} p^{\prime} ; p_{1}\right) \cap \bar{l}^{-}\left(q_{2}^{\prime} q_{3}^{\prime} ; p_{1}\right) \cap \bar{l}^{-}\left(q_{3}^{\prime} p^{\prime \prime} ; p_{1}\right) .
$$

The above discussion says that

$$
\begin{array}{llrl}
\overline{q_{2}^{\prime} q_{3}^{\prime}} \subset\left(\quad \bar{l}^{-}\left(p_{1} q_{2}^{\prime} ; p_{4}\right) \cap \bar{l}^{+}\left(q_{2}^{\prime} q_{3}^{\prime} ; p_{1}\right) \cap \bar{l}^{+}\left(p_{1} q_{3}^{\prime} ; q_{2}^{\prime}\right)\right), \\
M_{0}^{\prime} \subset\left(\bar{l}^{+}\left(p_{1} p_{4} ; p_{2}\right) \cap \bar{l}^{+}\left(q_{2}^{\prime} p^{\prime} ; p_{1}\right)\right. & \cap \bar{l}^{+}\left(p_{1} q_{2}^{\prime} ; p_{4}\right) & )-\left\{p_{4}, p^{\prime}\right\}, \\
M_{1}^{\prime \prime} \subset( & \bar{l}^{-}\left(q_{2}^{\prime} p^{\prime} ; p_{1}\right) \cap \bar{l}^{-}\left(q_{3}^{\prime} p^{\prime \prime} ; p_{1}\right) & \cap \bar{l}^{-}\left(q_{2}^{\prime} q_{3}^{\prime} ; p_{1}\right) & )-\left\{q_{3}^{\prime}\right\}, \\
M_{h}^{\prime \prime} \subset\left(\bar{l}^{-}\left(p_{1} p_{4} ; p_{2}\right)\right. & \cap \bar{l}^{+}\left(q_{3}^{\prime} p^{\prime \prime} ; p_{1}\right) & \cap \bar{l}^{-}\left(p_{1} q_{3}^{\prime} ; q_{2}^{\prime}\right)-\left\{p_{1}, p^{\prime \prime}\right\} .
\end{array}
$$

Then $M_{0}^{\prime}, M_{1}^{\prime \prime}, M_{h}^{\prime \prime}, q_{2}^{\prime}$, and $q_{3}^{\prime}$ satisfy conditions (3.5)-(3.12).

Lemmas 3.3, 3.4, 3.6, 3.7, and 3.8 indicate the existence of rt-embeddings of any rooted tree with $n$ vertices on any set of $n$ points in general position in the plane. An rt-embedding can be constructed of a given rooted tree on any given set of $n$ points. Moreover, from the discussion of this paper, we can do this in polynomial time with respect to $n$.

Theorem 3.9. Let $T$ be any rooted tree with $n$ vertices $V(T)$ and let $N$ be a set of $n$ points in $R^{2}$. If no three points of $N$ lie on any line, there is an rt-embedding of $V(T)$ on $N$. Furthermore, some rt-embedding can be constructed in polynomial time with respect to $n$. 


\section{Complexity}

In this section we discuss the time complexity of our algorithm for finding an rt-embedding. Our arguments are limited to overall results and we refer the reader to [4] and [1] for details. We assume that Algorithms 1 and 2 require $f(n)$ and $f^{\prime}(n)$ times, respectively. These have been analyzed to require $O\left(n^{2}\right)$ time in [2].

First we must reconstruct $T$ to satisfy condition (2.1) and enumerate the numbers $s$ and $t$. By using the postorder traversal for trees, we can modify $T$ in $O(n)$ time. To find the numbers $s$ and $t$, we first sort $N-\left\{p_{1}\right\}$ around $p_{1}$ in $O(n \log n)$ time. Then $s$ and $t$ can be found in $O(n)$ time. $O(n)$ time is also sufficient to find the master $r_{k}$ satisfying (3.1), and to determine whether a given instance belongs to Case 1,2 , or 3 . The procedure for Case 1 requires $O\left(n+\sum_{i=1}^{c h\left(r_{1}\right)} f\left(\left|T^{i}\left(r_{1}\right)\right|+1\right)\right)$ time because $N-\left\{p_{1}\right\}$ are already sorted around $p_{1}$. In the same way, an rt-embedding is found in $O\left(n+f(n)+f^{\prime}(n)\right)$ time in Case 2. In Case $3 M_{0}, M_{1}$, $M_{h}, p_{2}, p_{3}, p_{4}$, and $q_{2}$ are enumerated in $O(n)$ time. In constant time we can determine which among Cases 3.1, 3.2, and 3.3 holds. In Case 3.1 the time complexity is $O\left(2 f(n)+f^{\prime}(n)\right)$. In Case 3.2 we can enumerate $M_{1}^{\prime}$ and $M_{h}^{\prime}$ by selecting the $\left|M_{1}\right|$ th point $p$ in linear time, and, hence, an rt-embedding is found in $O\left(n+2 f(n)+f^{\prime}(n)\right)$ time. In Case 3.3 the sequence $\sigma$ of extreme points of $\operatorname{conv}\left(M_{1}\right)$ is found in linear time by using the incremental method and the clockwise ordering on $M_{1}$ around $p_{1}$. In the same way as Case $3.2, M_{0}^{\prime}, M_{1}^{\prime}$, and $p^{\prime}$ are found in linear time, and then $M_{1}^{\prime \prime}, M_{h}^{\prime \prime}$, and $p^{\prime \prime}$ are also found in linear time in Case 3.3(b). Hence an rt-embedding is found in $O\left(n+2 f(n)+f^{\prime}(n)\right)$ time in Case 3.3. To summarize the above discussion, the time complexity of our algorithm is $O\left(\max \left\{n \log n, \sum_{i=1}^{c h\left(r_{1}\right)} f\left(\left|T^{i}\left(r_{1}\right)\right|+1\right), f(n), f^{\prime}(n)\right\}\right)$. Hence the time complexity of our algorithm is $O\left(n^{2}\right)$, and obviously the space complexity is $O(n)$. We remark that any speedup in the computation when $p_{1}$ is an extreme point would immediately shorten the overall computation time.

\section{Acknowledgments}

We would like to thank Professor Imre Bárány for his kind advice, and the anonymous referee whose helpful comments improved the presentation of this paper.

\section{References}

1. Edelsbrunner, H. (1987), Algorithms in Combinatorial Geometry, Springer-Verlag, Berlin.

2. Pach, J., and Töröcsik, J. (1993), Layout of rooted trees, in Planar Graphs (W. T. Trotter, ed.), DIMACS Series, Vol. 9, American Mathematical Society, Providence, RI, pp. 131-137.

3. Perles, M. (1990), Open problem proposed at the DIMACS Workshop on Arrangements, Rutgers University.

4. Preparata, F. P., and Shamos, L. 1. (1985), Computational Geometry-An Introduction, SpringerVerlag, New York. 